\title{
Yaw Stability Control Design Through a Mixed-Sensitivity Approach
}

\author{
Vito Cerone, Member, IEEE, Mario Milanese, Senior Member, IEEE, and Diego Regruto, Member, IEEE
}

\begin{abstract}
In this paper, a vehicle dynamic control (VDC) system for tracking the desired vehicle behavior is developed. A 2-DOF control structure is proposed to prevent vehicle skidding during critical maneuvers through the application of differential braking between the right and left wheels in order to control yaw motion. The feedforward filter is a reference generator which computes the desired yaw rate on the basis of the steering angle, while the feedback controller is designed to track the reference as close as possible and to satisfy suitable loop robustness requirements. Mixedsensitivity minimization techniques are exploited in order to design the loop controller. The performance of the control system is evaluated through the hardware-in-the-loop simulation system under both emergency maneuvers and noncritical driving conditions, i.e., when the VDC system is not supposed to intervene.
\end{abstract}

Index Terms-Hardware-in-the-loop (HIL) simulation, robust control, vehicle dynamic control (VDC), vehicle lateral stability, yaw moment control.

\section{INTRODUCTION}

$\mathbf{R}$ OAD vehicles are generally equipped with passive and/or active safety systems. The main purpose of passive safety systems (e.g., seat belts and air bags) is to mitigate the severity of an accident. Active safety systems instead help prevent accidents by temporarily taking control away from the driver until the undesired vehicle dynamic behavior is corrected.

One of the most studied active safety systems which aims at enhancing the vehicle yaw stability is the vehicle dynamic control (VDC) system. Indeed, loss of vehicle yaw stability may result either from inappropriate driver's action or from unexpected yaw disturbances like side-wind force, tire pressure loss, or $\mu$-split braking due to unilaterally different road such as icy or wet pavement. Safe driving requires the driver to react rapidly and properly. Unfortunately, average drivers may exhibit panic reaction and may not be able to work out adequate steering and/or braking/throttle commands. The main goal of vehicle yaw stability control systems is to compensate for the driver's

Manuscript received March 16, 2007; revised December 03, 2007 and April 17, 2008. Manuscript received in final form June 26, 2008. First published August 11, 2009; current version published August 26, 2009. Recommended by Associate Editor C. Bohn. This work was supported in part by the Italian Ministero dell'Università e della Ricerca (MUR) under the plan "Control of advanced systems of transmission, suspension, steering and braking for the management of the vehicle dynamics" and in part by FIAT S.p.A. under the contract "Metodologie di impostazione e sviluppo algoritmi e strategie di controllo dell'autotelaio-Logiche di controllo innovative basate sulla frenatura dinamica."

The authors are with the Dipartimento di Automatica e Informatica, Politecnico di Torino, 10129 Torino, Italy (e-mail: vito.cerone@polito.it; mario.milanese@polito.it; diego.regruto@polito.it).

Color versions of one or more of the figures in this paper are available online at http://ieeexplore.ieee.org.

Digital Object Identifier 10.1109/TCST.2008.2005402 inadequacy and generate a control yaw moment through either steering or braking control inputs or both.

Yaw stability control systems have been established in the automotive industry as a safety/performance/comfort feature. They generally provide a control action which prevents the vehicle from under- or oversteering in a handling maneuver (e.g., lane change, slalom, etc.), particularly on a low-friction-coefficient surface. VDC systems directly control the yaw moment by generating differential longitudinal forces on the left and right tires, which, in turn, effectively affect the vehicle lateral motion. The two primary corrective yaw-moment-generating methods of actuation for VDC systems are compensation using steering commands or using differential wheel braking.

Pioneering results on VDC can be found in [1]-[3]. Most of the commercially available VDC systems use differential wheel braking as it is more easily accomplished through already existing ABS hardware (see, e.g., [4]). In [5], a four-wheel steering system with the use of yaw rate feedback and steering-angle feedforward control is investigated. When the vehicle becomes unstable because of sudden side wind, road surface disturbance, or abrupt braking, steering is automatically corrected through the rear wheel to improve stability.

In [6], a chassis control strategy for improving the limit performance of vehicle motion is proposed. The effects of braking force distribution on vehicle lateral and longitudinal directions are studied. A method to improve the handling and stability of vehicles by controlling the yaw moment generated by driving/braking forces is presented in [7]. Yaw moment is controlled by the feedforward compensation of steering angle and velocity to minimize the sideslip angle at the vehicle center of gravity. In [8], an integrated control system of active rear-wheel steering and yaw moment control using braking forces is presented. The control system is designed using model-matching control theory to make the vehicle performance follow a desired dynamic model. An $H_{\infty}$ yaw moment control using brake torque for improving vehicle performance and stability in high-speed driving is described in [9].

In [10], a 2-DOF steering controller architecture based on a disturbance observer method is adapted to the vehicle yaw dynamic problem and shown to robustly improve vehicle yaw dynamic performance. An auxiliary-steering actuation system, a steering controller that only intervenes when necessary, and a velocity-gain-scheduled implementation that is tested throughout the range of operation are considered. In [11], the predictive characteristics of the generalized predictive control are exploited in order to derive a yaw stability control algorithm. The control algorithm is based on a linearized vehicle model.

A VDC system for improving dynamic stability under critical lateral motions is developed in [12]. The use of yaw mo- 
ment control is investigated by adjusting the wheel slip ratio for improving the handling and stability of vehicle. The purpose of the proposed control system is to make the yaw rate and sideslip angle of the vehicle track their corresponding desired values.

In this paper, a VDC system for tracking the desired vehicle behavior is developed. A 2-DOF control structure is proposed to prevent vehicle skidding during critical maneuvers through the application of differential braking between the right and left wheels in order to control yaw motion. The feedforward filter is a reference generator which computes the desired yaw rate on the basis of the steering angle, while the feedback controller is designed to track the reference as close as possible and to satisfy suitable loop robustness requirements. The design of the loop controller and the identification of an approximate mathematical model of the plant that is used to design the controller are treated as a joint problem formulated as the minimization of a suitable mixed-sensitivity functional which depends on both the controller and the plant model. A suboptimal solution to such a problem is computed by exploiting a proper iterative algorithm whose initialization only requires a controller which stabilizes the closed-loop system even if it provides unacceptable performances. The main advantage of the proposed approach with respect to the available literature on the subject is that no accurate mathematical modeling of the vehicle is required to initialize the controller design procedure, since model identification and controller design are jointly solved.

\section{PlANT DESCRIPTION}

The plant to be controlled is a hardware-in-the-loop (HIL) test bench built by the Vehicle Dynamics Research Team, Mechanical Engineering Department, Politecnico di Torino, Torino, Italy [13], [14]. Such a test bench, as shown in Fig. 1, consists of the whole braking system of a FIAT passenger car properly interfaced, through a personal computer and a suitable dSPACE board, with a real-time vehicle dynamic simulator. More precisely, the components of the system are the vacuum booster, the tandem master cylinder, the wheel calipers with their mechanical support, and a hydraulic VDC unit with 12 PWM-controlled solenoid valves used to regulate the oil pressure in the brake chambers. The hydraulic circuit of the test bench consists of an electric motor, a gear pump, an accumulator, some pressure limiter/reducer valves, and a proportional valve. Four pressure sensors are used to measure the four wheel caliper pressures which are the inputs of the vehicle model. From such measurements, wheel braking torques are easily computed by exploiting standard physical equations (see, e.g., [15]).

The real-time nonlinear model used on the test bench to simulate the vehicle dynamics has 8 DOF. Four degrees (lateral, longitudinal, yaw, and roll motions) account for the vehicle body dynamics, while four degrees are used to describe the wheel rotational motions. Tires were modeled using the Pacejka magic formula [16].

The test bench also includes a low-level open-loop controller of the hydraulic actuator. Such a controller suitably regulates both the PWM voltage of the solenoid valves and the hydraulic pump on/off signal in order to actuate the desired pressure signal provided by the high-level VDC controller to be designed.

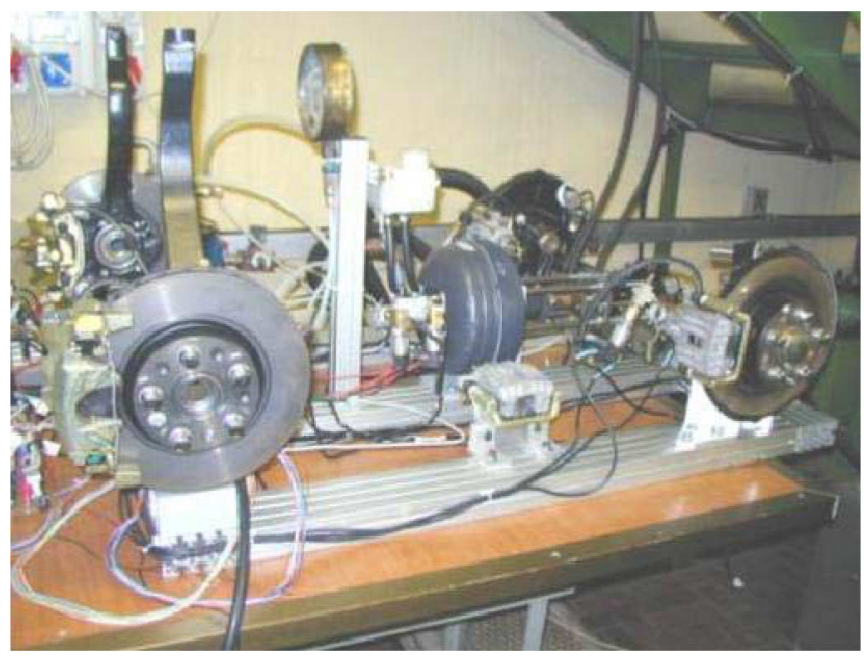

Fig. 1. HIL test bench.

A set of different maneuvers can be performed by means of a suitable driver simulator included in the model. In [14], a validation of the overall vehicle model is performed. Simulated and experimental results are compared on a suitable range of maneuvers, including steering-angle steps, ramps, and frequency sweep. A detailed description of the test bench can be found in the papers by Sorniotti [13] and Sorniotti and Velardocchia [14].

\section{Control ObJective AND Performance ReQuirements}

The problem that we are dealing with in this paper is the design of a closed-loop control system that is able to prevent vehicle skidding during critical maneuvers through the application of differential braking between the right and left wheels in order to control yaw motion. More specifically, the control problem is defined with reference to the following two specific tests:

1) Step steer test: The test starts with a vehicle traveling at the constant speed of $100 \mathrm{~km} / \mathrm{h}$. The driver turns the steering wheel at the speed of $250^{\circ} / \mathrm{s}$ until a specified target angle is reached. Then, the target angle is held. Different steering target angles have to be considered from $50^{\circ}$ to $110^{\circ}$. This maneuver is considered in order to define the desired behavior of the controlled vehicle in critical driving conditions.

2) Slow ramp steer test: The test is performed at the constant speed of $100 \mathrm{~km} / \mathrm{h}$. The driver slowly increases the steering wheel angle $\left(15^{\circ} / \mathrm{s}\right)$ from $0^{\circ}$ to $130^{\circ}$. This maneuver is used to define the desired behavior of the controlled vehicle in noncritical driving conditions.

The following symbols are introduced in order to formulate a set of performance requirements:

$t_{0} \quad$ starting time of the maneuver;

$\dot{\psi}_{c} \quad$ controlled-vehicle yaw rate;

$\dot{\psi}_{u} \quad$ uncontrolled-vehicle yaw rate;

$a_{y_{c}} \quad$ controlled-vehicle lateral acceleration;

$a_{y_{u}} \quad$ uncontrolled-vehicle lateral acceleration; 


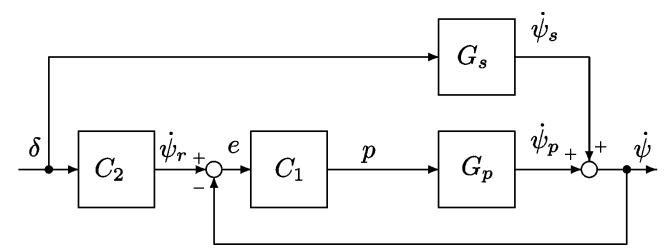

Fig. 2. Block diagram of the considered control system.

$$
\begin{aligned}
& \beta \quad \text { vehicle sideslip angle; } \\
& \dot{\psi}_{c}^{\star} \quad \text { amplitude of the first local yaw rate extremum } \\
& \text { (maximum/minimum); } \\
& \dot{\psi}_{c}^{\circ} \quad \text { amplitude of the second local yaw rate extremum } \\
& \text { (minimum/maximum); } \\
& t^{\star} \quad \text { time instant at which the first local yaw rate } \\
& \text { extremum occurs }\left(t^{\star}=\min \left\{t: \dot{\psi}_{c}(t)=\right.\right. \\
& \left.\left.\dot{\psi}_{c}^{\star}, \ddot{\psi}(t)=0\right\}\right) \text {; }
\end{aligned}
$$

FIAT S.p.A. provided a list of specific requirements reported here as a set of five performance specifications which have to be satisfied by the controlled vehicle when a step steer test is run:

1) time between the first and second peaks of yaw rate of less than $1 \mathrm{~s}:\left|t^{\star}-t^{\circ}\right|<1 \mathrm{~s}$

2) amplitude difference between the first and second peaks of yaw rate of less than $10^{\circ} / \mathrm{s}:\left|\dot{\psi}_{c}^{\star}-\dot{\psi}_{c}^{\circ}\right|<10^{\circ} / \mathrm{s}$;

3) sideslip-angle peak amplitude of less than $7^{\circ} / \mathrm{s}$ : $\max _{t}|\beta(t)|<7^{\circ}$;

4) difference between controlled- and passive-vehicle yaw rates of less than $1^{\circ} / \mathrm{s}$ when $t<t_{0}+0.8 \mathrm{~s}:\left|\dot{\psi}_{c}-\dot{\psi}_{u}\right|<$ $1^{\circ} / \mathrm{s} \forall t \in\left[t_{0}, t_{0}+0.8 \mathrm{~s}\right]$;

5) difference between controlled- and passive-vehicle steady-state lateral accelerations of less than $0.2 \mathrm{~m} / \mathrm{s}^{2}$ : $\lim _{t \rightarrow \infty}\left|a_{y_{c}}-a_{y_{u}}\right|<0.2 \mathrm{~m} / \mathrm{s}^{2}$;

6) difference between controlled- and passive-vehicle longitudinal velocities of less than $5 \mathrm{~km} / \mathrm{h}$ : $\max _{t} \mid v_{x_{u}}(t)-$ $v_{x_{c}}(t) \mid<5 \mathrm{~km} / \mathrm{h}$.

All specifications [1)-6)] have to be satisfied for each value of the step amplitude between $50^{\circ}$ and $110^{\circ}$.

As far as the ramp steer test is considered, the controller is required to not act on the braking system during such a maneuver in order to avoid perturbation of the behavior of the car during noncritical driving conditions.

\section{Control Structure AND Strategy}

\section{A. Control Structure}

In order to meet the performance requirements specified in Section III, the 2-DOF control structure of Fig. 2 is proposed, where controller $C_{2}$ is a reference generator which computes the desired yaw rate on the basis of the steering angle, and $C_{1}$ is the feedback controller that is designed to track the reference as well as possible while satisfying suitable loop robustness requirements.

The output signal of controller $C_{1}$ is the plant command input $p(t)$. The absolute value $|p(t)|$ is the actual reference oil pressure to be actuated in the brake chamber while the sign of $p(t)$ is used, together with the sign of the steering angle $\delta(t)$, to select the wheel brake that has to be activated. More specifically, a brake caliper selector acts on the hydraulic circuit to apply the pressure $|p(t)|$ to either the right- or left-wheel brakes according to the following rule: Left-wheel brakes are activated if either $\delta(t), p(t)>0$ or $\delta(t), p(t)<0$; right-wheel brakes are activated otherwise.

The vehicle is modeled by $G_{s}(s)$, i.e., the transfer function between the steering angle $\delta$ and the yaw rate $\dot{\psi}$, and by $G_{p}(s)$ which is a linear model of the relation between the desired pressure $p(t)$ and the yaw rate, including the controlled actuator and the caliper selector. The transfer function $G_{s}(s)$ has been obtained from the standard bicycle model (see, e.g., [15]) assuming the steering-wheel angle as input, the vehicle sideslip angle and the yaw rate as state variables, and the yaw rate as output. The transfer function $G_{s}(s)$ written in terms of the vehicle physical parameters is given by

$$
G_{s}=\frac{b_{0}+b_{1} s}{a_{0}+a_{1} s+a_{2} s^{2}}
$$

where

$$
\begin{aligned}
b_{0} & =\frac{c_{f} c_{r}\left(l_{f}+l_{r}\right) v}{r_{\delta}} \\
b_{1} & =\frac{c_{f} l_{f} m v^{2}}{r_{\delta}} \\
a_{0} & =c_{f} c_{r}\left(l_{f}+l_{r}\right)^{2}+\left(c_{r} l_{r}-c_{f} l_{f}\right) m v^{2} \\
a_{1} & =\left(c_{f}\left(J_{z}+l_{f}^{2} m\right)+c_{r}\left(J_{z}+l_{r}^{2} m\right)\right) v \\
a_{2} & =J_{z} m v^{2} .
\end{aligned}
$$

The symbol $m$ denotes the vehicle mass; $J_{z}$ is the moment of inertia around the vertical axis; $l_{f}$ and $l_{r}$ are the distances between the vehicle center of gravity and the front and rear axles, respectively; $v$ is the vehicle speed; and $r_{\delta}$ is the ratio between the steering-wheel angle and the front-wheel angle. The parameters $c_{f}$ and $c_{r}$ are the so-called front and rear cornering stiffness coefficients, respectively, which are used to describe the tire behavior.

A linear model of the tire has been assumed, where the lateral tire force is described as the product of the cornering stiffness and the wheel sideslip angle (see, e.g., [15]). The values of the cornering stiffness coefficients $c_{f}$ and $c_{r}$ have been properly tuned in order to get the best matching between the simulated response of model $G_{s}(s)$ and the experimental yaw rate response of the passive vehicle in critical driving conditions. The values of all the physical vehicle parameters are summarized in Table I. The transfer function (1) has been obtained by setting $v=100 \mathrm{~km} / \mathrm{h}$. How to obtain model $G_{p}(s)$ will be discussed in Section IV-E.

\section{B. Design of the Reference Generator $C_{2}$}

In order to properly design the reference generator $C_{2}$, it is first required to define the main properties of the desired 
TABLE I

VALUES OF MODEL PARAMETERS

\begin{tabular}{|c|c|}
\hline Parameter & value \\
\hline$m(\mathrm{~kg})$ & 1678 \\
\hline$J_{z}\left(\mathrm{kgm}^{2}\right)$ & 3070 \\
\hline$c_{r}(\mathrm{~N} / \mathrm{rad})$ & 37425 \\
\hline$c_{f}(\mathrm{~N} / \mathrm{rad})$ & 28648 \\
\hline$l_{r}(\mathrm{~m})$ & 1.55 \\
\hline$l_{f}(\mathrm{~m})$ & 1.15 \\
\hline$r_{\delta}$ & 13.04 \\
\hline
\end{tabular}

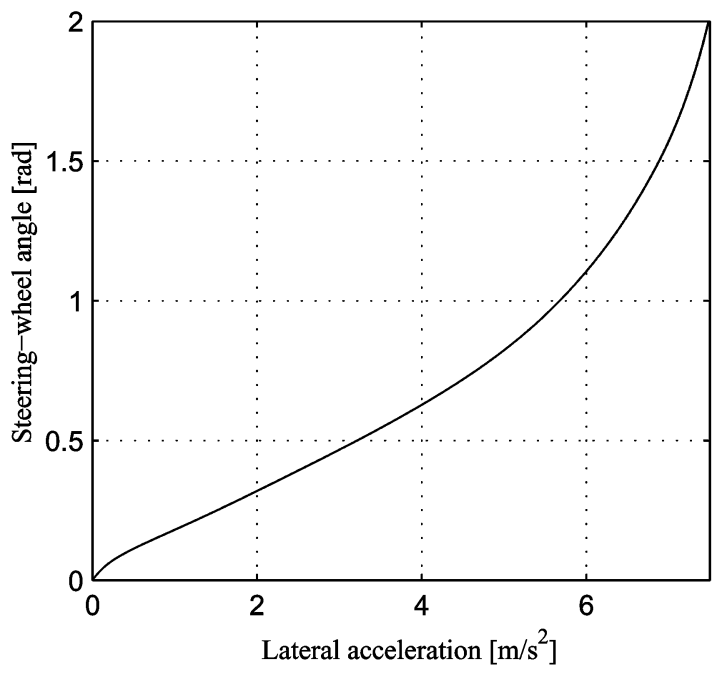

Fig. 3. Vehicle steering diagram considered in this paper corresponding to $v_{x}=100 \mathrm{~km} / \mathrm{h}$.

yaw rate to be generated. Analysis of performance specifications leads to the choice of a yaw rate reference signal which has to preserve the steady-state behavior [specification 5)] and the speed of response [specification 4)] of the uncontrolled vehicle while significantly reducing the oscillation during transients [specifications 1)-3)]. In addition, the yaw rate reference signal generated when the driver performs a slow ramp steer test should be as close as possible to the yaw rate of the uncontrolled vehicle in order to guarantee that the control system will not act on the braking system.

The idea exploited in this paper to meet all such requirements is to compute the reference yaw rate $\dot{\psi}_{r}$ through the following convolution integral:

$$
\dot{\psi}_{r}(t)=f(t) * g(t)=f(t) *\left(h^{-1}\left(\delta(t), v_{x}(t)\right) / v_{x}(t)\right)
$$

where $f(t)$ is the impulse response of a low-pass filter with transfer function $F(s)=10 /(s+10)$ and the function $h\left(a_{y}, v_{x}(t)\right)$ is the so-called steering diagram of the uncontrolled vehicle which, for any fixed velocity $v_{x}(t)$, relates the lateral acceleration $a_{y}(t)$ and the steering angle $\delta(t)$ at steady state.

The steering diagram $\delta(t)=h\left(a_{y}(t), v_{x}(t)\right)$ of the vehicle considered in this paper is shown in Fig. 3 for the case of $v_{x}=$ $100 \mathrm{~km} / \mathrm{h}$. Since it is easy to show that, at constant longitudinal velocity $v_{x}$, the lateral acceleration $a_{y}(t)$ and the yaw rate $\dot{\psi}$ at steady state satisfy equation $a_{y}(t)=v_{x} \dot{\psi}(t)$ (see, e.g., [15eq. (8.26)]), it turns out that function $g(t)$ is, for any approximately constant velocity $v_{x}$, a good approximation of the static mapping which relates the steering angle $\delta(t)$ and the yaw rate $\dot{\psi}(t)$ of the passive vehicle at steady state. Thus, the proposed reference yaw rate, which, at steady state, equals $g(t)$, preserves the steady-state behavior of the vehicle [specification 5)].

The filter $F(s)$ was introduced to properly set the rise time of the controlled vehicle in order to obtain the same speed of response of the uncontrolled one [specification 4)]. Moreover, since $\dot{\psi}_{r}$ is the output of a static function filtered through a linear system with a real pole, the reference yaw rate will not show any oscillation when a step steer test is performed [specifications 1)-3)].

Finally, one must consider that the steering diagram $h\left(a_{y}(t), v_{x}(t)\right)$ is usually obtained by experimentally performing a slow ramp steer test. Thus, at least in principle, the proposed yaw rate reference will equal the actual yaw rate of the uncontrolled vehicle when a slow ramp steer test is performed, intrinsically avoiding the actuation of the braking system.

\section{Design of Controller $C_{1}(s)$ : Problem Formulation}

Feedback controller $C_{1}$ must be designed to track, as close as possible, the desired yaw rate provided by controller $C_{2}$ while guaranteeing satisfactory loop robustness margins. From the block diagram of Fig. 2, it is easily seen that the transfer function between the steering angle $\delta(t)$ and the tracking error $e(t)=\dot{\psi}_{r}-\dot{\psi}$ is given by

$$
\frac{E\left(s, C_{1}\right)}{\Delta(s)}=S\left(s, C_{1}\right)\left(\bar{C}_{2}(s)-G_{s}(s)\right)
$$

where $E\left(s, C_{1}\right)$ and $\Delta(s)$ are the Laplace transforms of the signals $e\left(t, C_{1}\right)$ and $\delta(t)$, respectively, $S(s)=1 /\left(1+C_{1}(s) G_{p}(s)\right)$ is the sensitivity function, and $\bar{C}_{2}(s)$ is a linear transfer function which approximates the nonlinear reference generator $C_{2}$.

Equation (4) shows that the tracking error $e(t)$ can be attenuated by properly shaping the frequency response of the sensitivity function $S(s)$. More precisely, our objective is to design a controller $C_{1}(s)$ such that the transfer function between the steering angle and the tracking error satisfies the following inequality:

$$
\left|\frac{E\left(j \omega, C_{1}\right)}{\Delta(j \omega)}\right| \leq\left|W^{-1}(j \omega)\right| \quad \forall \omega
$$

where $W(s)$ is a rational function which properly embeds tracking performance requirements.

From (4) and (5), and the definition of $H_{\infty}$ norm of a singleinput-single-output system, the control problem can be formulated as the following $H_{\infty}$ sensitivity minimization problem:

$$
C_{1}^{*}(s)=\arg \min _{C_{1} \in \mathcal{C}}\left\|S\left(s, C_{1}\right) W_{S}(s)\right\|_{\infty}
$$

where $\mathcal{C}$ is the class of controllers which stabilizes the plant and $\left|W_{S}(j \omega)\right| \geq\left|W(j \omega)\left(\bar{C}_{2}(j \omega)-G_{s}(j \omega)\right)\right| \forall \omega$. As is well known, a specification of the kind $\left\|S\left(s, C_{1}\right) W_{S}(s)\right\|_{\infty}<1$ provides a constraint on the steady-state behavior of the controlled system and can also force a lower bound on its bandwidth, while it does not constraint the bandwidth from above 
(see, e.g., [17Ch. 2]). Thus, the solution of a pure weighted-sensitivity minimization problem could lead to the design of a control system with possible undesired large bandwidth which, in turn, could cause instability problems when the controller is applied to the real plant due to the presence of significant high-frequency model uncertainty [17]. In order to limit the control system bandwidth, controller $C_{1}(s)$ is actually computed by solving the following mixed-sensitivity problem:

$$
\begin{aligned}
C_{1}^{*}(s) & =\arg \min _{C_{1} \in \mathcal{C}} J\left(C_{1}, G_{p}\right) \\
& =\arg \min _{C_{1} \in \mathcal{C}}\left\|\begin{array}{c}
S\left(C_{1}, G_{p}\right) W_{S}(s) \\
T\left(C_{1}, G_{p}\right) W_{T}(S)
\end{array}\right\|_{\infty}
\end{aligned}
$$

where $T\left(C_{1}, G_{p}\right)=C_{1} G_{p} /\left(1+C_{1} G_{p}\right)$ is the complementary sensitivity function and where $W_{T}(s)$ is a rational function which properly embeds bandwidth requirements.

\section{Design of Controller $C_{1}(s)$ : Selection of Weighting}

Functions $W_{S}(s)$ and $W_{T}(s)$

The following structure has been assumed for the weighting function $W_{S}(s)$ :

$$
W_{S}(s)=\frac{s^{2} / \omega_{n}^{2}+1.414 s / \omega_{n}+1}{\alpha(s / z+1) s}
$$

where the pole at the origin has been included to guarantee robust tracking of constant signal, and parameters $z, \omega_{n}$, and $\alpha$ must be selected in order to provide a lower bound on the control system bandwidth and to satisfy the inequality $\left|W_{S}(j \omega)\right| \geq$ $\left|W(j \omega)\left(\bar{C}_{2}(j \omega)-G_{s}(j \omega)\right)\right| \forall \omega$. The values $z=0.8, \alpha=$ 0.12 and $\omega_{n}=3$ have been selected.

The weighting function $W_{T}(s)=(s / 10+1) / 1.2$ has been chosen in order to force a proper upper bound on the control system bandwidth. The transfer function $G_{s}(s)=0.82307(s+1.885) /\left(s^{2}+2.916 s+10.13\right)$ has been obtained from the linear bicycle model presented in Section IV-A. The linear model $\bar{C}_{2}(s)=1.636 /(s+10)$ of the reference generator has been obtained by approximating the steering diagram at velocity $v_{x}=100$ with a constant gain.

\section{E. Design of Controller $C_{1}(s)$ : Iterative Approach}

The computation of the controller as a solution of problem (7) is based on a model $G_{p}(s)$ which is an approximate description of the true unknown plant, including also the dynamics of the hydraulic actuator. Thus, the performance of the actual control system will depend on such a model, and the problem on how to properly select the transfer function $G_{p}(s)$ arises.

As pointed out in [18] and [19], approximate model identification and model-based controller design have to be treated as a joint problem in order to guarantee a reasonable degree of robustness. To be more precise, let us use the symbol $\tilde{G}_{p}$ to indicate the unknown true plant. The actual problem to be solved can be formulated as

$$
\begin{aligned}
C_{1}^{*}(s) & =\arg \min _{C_{1} \in \mathcal{C}} J\left(C_{1}, \tilde{G}_{p}\right) \\
& =\arg \min _{C_{1} \in \mathcal{C}}\left\|\begin{array}{c}
S\left(C_{1}, \tilde{G}_{p}\right) W_{S}(s) \\
T\left(C_{1}, \tilde{G}_{p}\right) W_{T}(S)
\end{array}\right\|_{\infty}
\end{aligned}
$$

where $J\left(C_{1}, \tilde{G}_{p}\right)$ is the mixed-sensitivity functional of the actual control system. Since the true plant $\tilde{G}_{p}$ is not known, problem (9) cannot be exactly solved. However, according to [18] and [19], we can consider the following triangle inequality:

$$
\begin{aligned}
\left\|J\left(C_{1}, \tilde{G}_{p}\right)\right\|_{\infty} \leq \| J( & \left.C_{1}, G_{p}\right) \|_{\infty} \\
& +\left\|J\left(C_{1}, \tilde{G}_{p}\right)-J\left(C_{1}, G_{p}\right)\right\|_{\infty} .
\end{aligned}
$$

Exploiting (10), the control problem can be reformulated as the following minimization problem:

$$
\begin{aligned}
& \left(C_{1}^{*}(s), G_{p}^{*}(s)\right) \\
& =\arg \min _{C_{1}, G_{p}}\left\{\left\|J\left(C_{1}, G_{p}\right)\right\|_{\infty}+\left\|J\left(C_{1}, \tilde{G}_{p}\right)-J\left(C_{1}, G_{p}\right)\right\|_{\infty}\right\} .
\end{aligned}
$$

In order to solve problem (11), an iterative approach has been proposed in [18] and [19]. At the $i$ th iteration, the plant model $G_{p}^{i}(s)$ and the feedback controller $C_{1}^{i}(s)$ are computed according to

$$
\begin{aligned}
& G_{p}^{i}(s)=\arg \min _{G_{p}}\left\|J\left(C_{1}^{i-1}, \tilde{G}_{p}\right)-J\left(C_{1}^{i-1}, G_{p}\right)\right\|_{\infty} \\
& C_{1}^{i}(s)=\arg \min _{C_{1}}\left\|J\left(C_{1}, G_{p}^{i}\right)\right\|_{\infty} \cdot
\end{aligned}
$$

Problem (12) is equivalent to an $H_{\infty}$ closed-loop identification problem and can be solved by exploiting the approach proposed in [20] which is based on the dual Youla parameterization of all plants that are stabilized by a given controller.

The identification is performed by exploiting the experimental closed-loop data obtained through the implementation of controller $C_{1}^{i-1}$ on the test bench. More specifically, the model $G_{p}^{i}$ is identified using the variables $p$ and $\dot{\psi}_{p}$ (see Fig. 2) which are the input and the output of $G_{p}$, respectively. The command input $p$ is physically measured on the test bench, while the output $\dot{\psi}_{p}$ is computed as $\dot{\psi}_{p}=\dot{\psi}-\dot{\psi}_{s}$, where $\dot{\psi}$ is the vehicle yaw rate measurement and $\psi_{s}$ can be easily computed as the output of model $G_{s}$, i.e., $\dot{\psi}_{s}=g_{s}(t) * \delta(t)\left(g_{s}(t)\right.$ is the impulse response of $G_{s}(s)$, while $*$ is the convolution operator). The algorithm proposed in [20] provides both the model $G_{p}^{i}(s)$ and the bound $W_{G_{p}^{i}}$ on the modeling error $\left|\tilde{G}_{p}-G_{p}^{i}\right|$ between the model and the true plant.

Problem (13) can be solved by means of standard $H_{\infty}$ control techniques (see, e.g., [17]) seeking for $C_{1}^{i}(s)$ among the classes of controllers which robustly stabilize the system $G_{p}^{i}(s)$ in the presence of additive unstructured uncertainty bounded by $W_{G_{p}^{i}}$. Thus, the obtained controller is a strictly proper transfer function whose order equals the sum of the orders of the plant and the weighting functions $W_{S}$ and $W_{T}$.

Remark: In the closed-loop identification stage [problem (12)], a model $G_{p}$ of the plant is identified by minimizing the error between the value of the closed-loop performance index obtained with the true plant $\tilde{G}_{p}$ and the value of the closed-loop performance index computed using the model $G_{p}$ (the interested reader can find details on the identification algorithm in [20]). The iterative procedure stops when the controlled system obtained by implementing controller $C_{i}(s)$ on the test bench satisfies the prescribed closed-loop performance requirements 


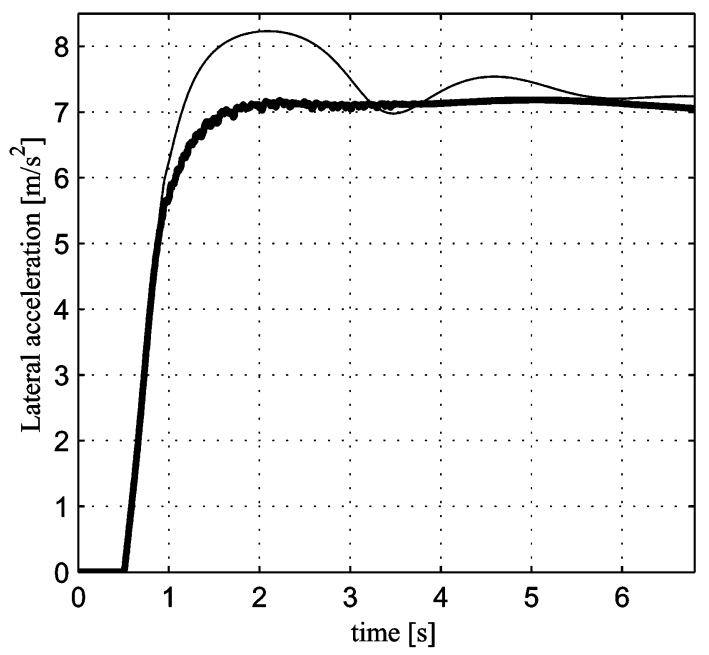

Fig. 4. Lateral acceleration: (Thick) Controlled vehicle and (thin) uncontrolled vehicle.

(note that the controller is implemented on the test bench at each iteration in order to collect data for closed-loop identification of $G_{p}$ ). Thus, in this procedure, a model $G_{p}$ is considered a good model for the true plant if the control system designed on the basis of such a model satisfies the performance requirements (even if the model $G_{p}$ is not quite close to the true plant).

Finally, we point out that no accurate model $G_{p}(s)$ of the dynamics of the vehicle equipped with a hydraulic actuator is needed to initialize the iterative design procedure, since the model identification problem and the controller design problem are jointly solved. This is the main advantage of the proposed approach with respect to the available literature on the subject.

\section{EXPERIMENTAL RESULTS AND DISCUSSION}

In this section, we report the experimental results obtained by testing the controlled system on the HIL test bench. A step steer test of $110^{\circ}$ on a dry road was performed first. The behavior of the vehicle during such a maneuver is highly nonlinear since, as shown in Fig. 4, the lateral acceleration approaches its saturation limit (see the steering diagram of Fig. 3). After three iterations, the following results have been obtained:

$$
\begin{aligned}
G_{p}^{3}(s) & =6.44 \cdot 10^{-3} \frac{(s+17.4)}{(s+7.745)(s+1.203)} \\
C_{1}^{3}(s) & =1.2 \cdot 10^{7} \frac{(s+7.745)(s+1.657)(s+1.203)}{s(s+6579)(s+17.82)(s+0.8)} .
\end{aligned}
$$

Controller $C_{1}^{3}$ largely satisfies performance specifications 1)-6), as shown by the experimental results presented in this section.

The yaw rate, the sideslip angle, and the vehicle velocity of the controlled and uncontrolled vehicles are compared in Figs. 5-7, respectively. Such figures show the effectiveness of the proposed control system which improves the performance of the uncontrolled vehicle and satisfies all specifications 1)-6). More specifically, the yaw rate of the controlled vehicle actually shows only one peak, implicitly satisfying both specifications 1 ) and 2). Furthermore, as clearly shown in Fig. 8, specification 4) is also satisfied since the maximum value of the difference between controlled- and passive-vehicle yaw rates is about $0.5^{\circ} / \mathrm{s}$

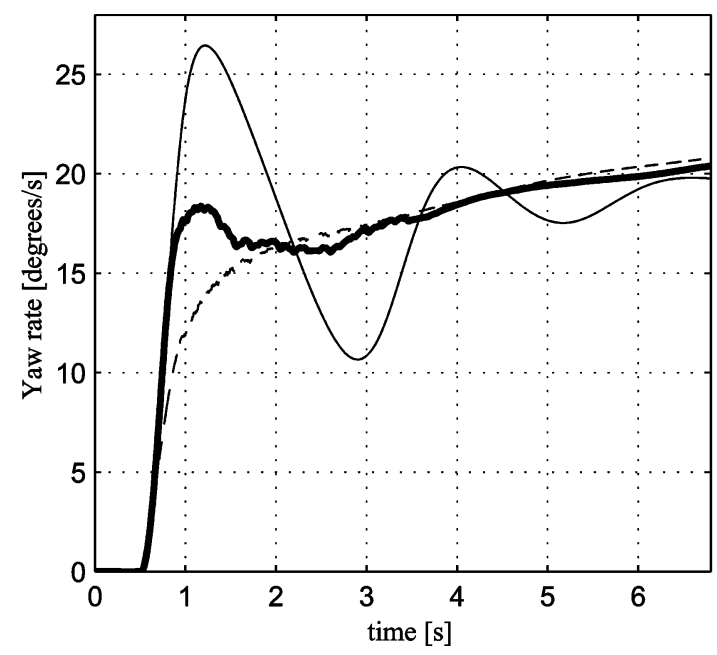

Fig. 5. Yaw rate response to a step steer test of $110^{\circ}$ : (Thick) Controlled vehicle, (thin) uncontrolled vehicle, and (dashed) reference.

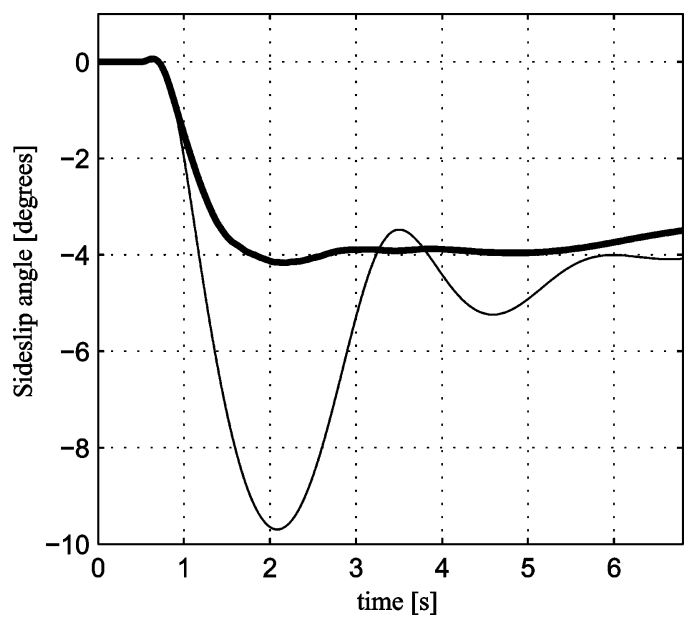

Fig. 6. Sideslip-angle response to a step steer test of $110^{\circ}$ : (Thick) Controlled vehicle and (thin) uncontrolled vehicle.

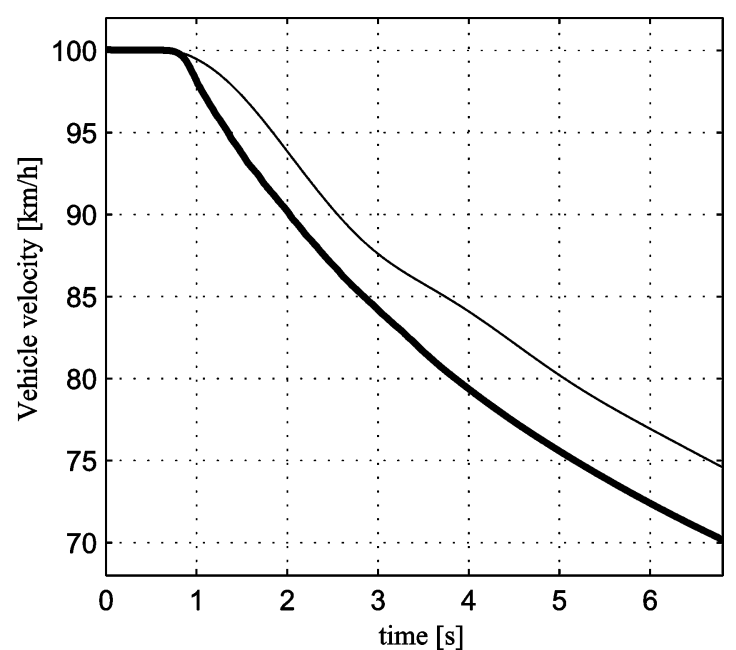

Fig. 7. Vehicle velocity: (Thick) Controlled vehicle and (thin) uncontrolled vehicle.

for $t<0.8 \mathrm{~s}$. Looking at Fig. 9, it can be seen that the difference between controlled- and passive-vehicle steady-state lateral accelerations is less than $0.2 \mathrm{~m} / \mathrm{s}^{2}$ for $t \geq 5.5 \mathrm{~s}$, showing the 


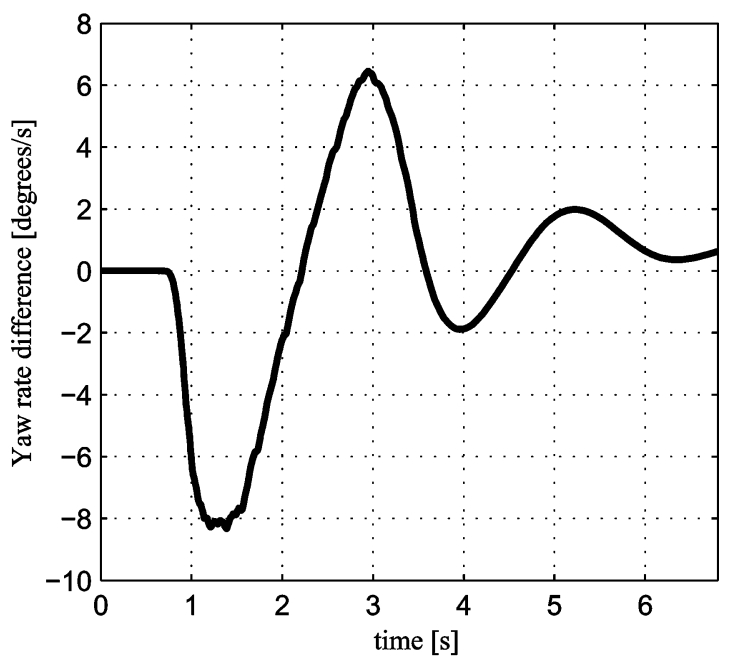

Fig. 8. Difference between controlled- and uncontrolled-vehicle yaw rates $\left(\dot{\psi}_{c}-\dot{\psi}_{u}\right)$.

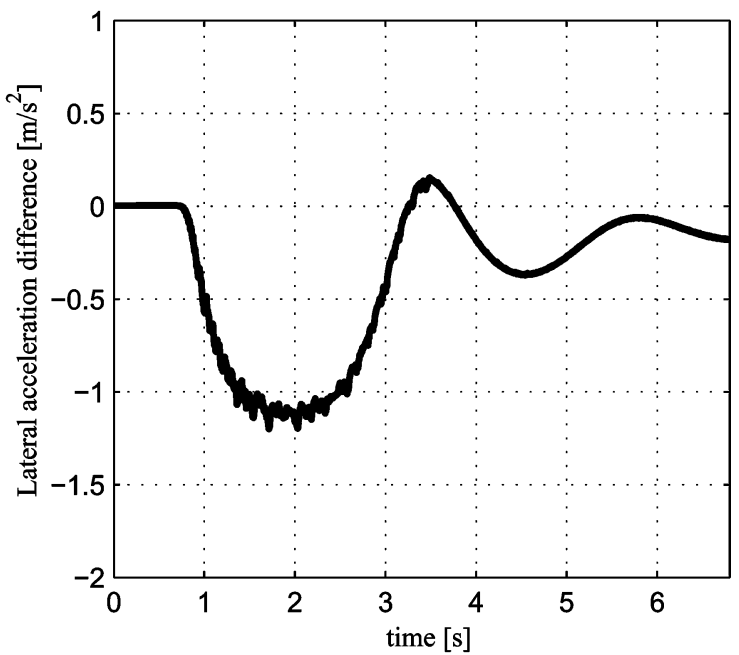

Fig. 9. Difference between controlled- and uncontrolled-vehicle lateral accelerations $\left(a_{y_{c}}-a_{y_{u}}\right)$.

fulfillment of specification 5). As far as specification 3) is considered, the absolute value of the sideslip-angle peak amplitude of the controlled vehicle is $4.2^{\circ}$, which is about $30 \%$ less than the maximum allowed one. Finally, as can be seen from Fig. 7 , the maximum difference between controlled- and passive-vehicle velocities is about $4.5 \mathrm{~km} / \mathrm{h}$; thus, specification 6) is also satisfied.

As discussed in Section IV-B, in principle, the yaw rate reference signal should be equal to the actual yaw rate of the uncontrolled vehicle when a slow ramp steer test is performed, intrinsically avoiding the actuation of the braking system. Since, in practice, the difference between the two signals is not exactly zero, it was necessary to introduce a threshold on the error signal $e(t)$. The control system is activated when the absolute value of the error is greater than $2^{\circ} / \mathrm{s}$.

In order to test the robustness of the designed control system, some additional maneuvers have been performed on the HIL test bench. Fig. 10 shows the comparison between the yaw rate of the controlled vehicle and the yaw rate of the uncontrolled one

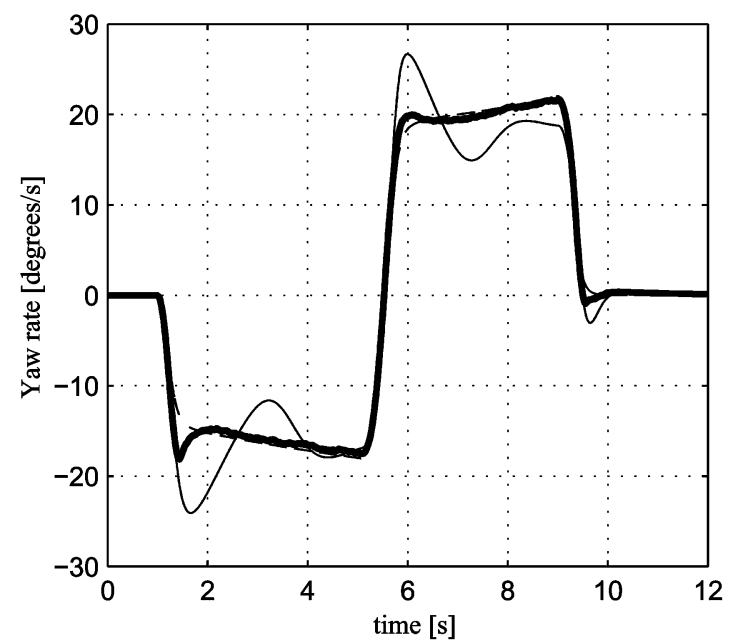

Fig. 10. Yaw rate response to a steer reversal test of $90^{\circ}$ : (Thick) Controlled vehicle, (thin) uncontrolled vehicle, and (dashed) reference.

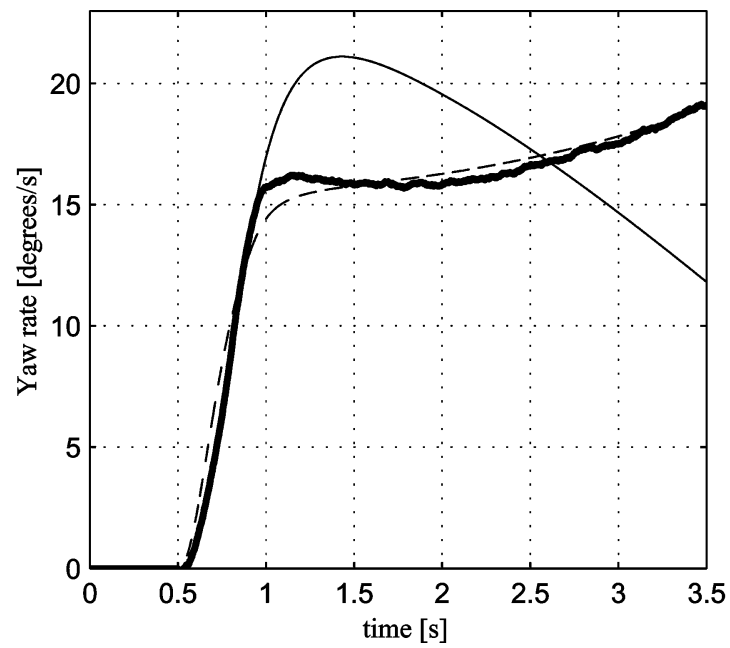

Fig. 11. Yaw rate response to a step steer test of $110^{\circ}$ that is performed on a wet road: (Thick) Controlled vehicle, (thin) uncontrolled vehicle, and (dashed) reference.

for a steer reversal test. The test starts with a vehicle traveling at the constant speed of $100 \mathrm{~km} / \mathrm{h}$. At time $t=1 \mathrm{~s}$, the driver turns the steering wheel at the speed of $250^{\circ} / \mathrm{s}$ until the angle of $-90^{\circ}$ is reached. Then, the target angle is held for $4 \mathrm{~s}$. At time $t=5 \mathrm{~s}$, the driver turns the steering wheel at the speed of $250^{\circ} / \mathrm{s}$ until the angle of $90^{\circ}$ is reached. Finally, after $4 \mathrm{~s}$, the steering-wheel angle is brought back to $0^{\circ}$.

Fig. 11 shows the comparison between the yaw rate of the controlled vehicle and the yaw rate of the uncontrolled one for a step steer test of $110^{\circ}$ that is performed on a wet road (adhesion coefficient $\mu=0.5$ ).

Finally, in order to test the disturbance rejection properties of the designed controlled systems, the effect of a side-wind gust acting on a vehicle that is driven along a straight road at the velocity of $100 \mathrm{~km} / \mathrm{h}$ has been considered. According to [21], a side wind impacting the car at the velocity $v_{w}$ exerts a force $F_{w}$ and a moment $M_{w}$, respectively, given by

$$
F_{w}=\frac{2.5 \pi}{2} v_{w}^{2}
$$




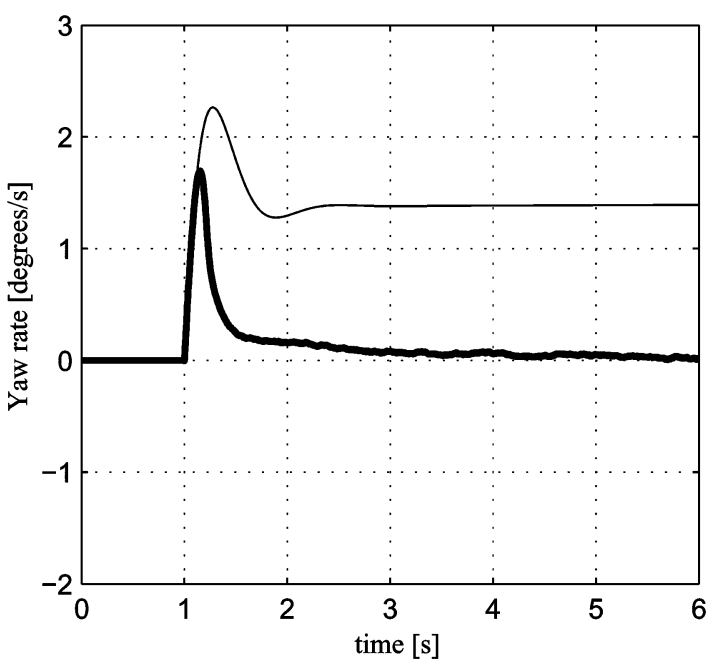

Fig. 12. Yaw rate response to a side-wind gust disturbance: (Thick) Controlled vehicle and (thin) uncontrolled vehicle.

$$
M_{w}=\left(2.5 \frac{\pi}{2}-3.3\left(\frac{\pi}{2}\right)^{3}\right) v_{w}^{2}+\frac{a-b}{2} F_{w} .
$$

The comparison between the response of the yaw rate of the controlled vehicle and the yaw rate of the uncontrolled one in the presence of a side-wind gust is shown in Fig. 12. The wind velocity $v_{w}$ equals zero until $t=1 \mathrm{~s}$ and value $v_{w}=10 \mathrm{~m} / \mathrm{s}$ from time $t=1 \mathrm{~s}$ on. All the presented maneuvers show that the proposed control system strongly improved the lateral behavior of the vehicle.

\section{CONCLUDING REMARKS}

In this paper, a 2-DOF control structure has been proposed for yaw stability control of a passenger car. The feedforward filter is a reference generator which computes the desired yaw rate on the basis of the steering angle, while the feedback controller is designed to track the reference as close as possible and to satisfy suitable loop robustness requirements.

The design of the loop controller and the identification of an approximate mathematical model of the plant that is used to design the controller have been treated as a joint problem formulated as the minimization of a suitable mixed-sensitivity functional which depends on both the controller and the plant model. A suboptimal solution to such a problem has been computed by exploiting a proper iterative algorithm whose initialization only requires a controller which stabilizes the closed-loop system even if it provides unacceptable performances. The main advantage of the proposed approach with respect to the available literature on the subject is that no accurate mathematical modeling of the vehicle is required to initialize the controller design procedure, since model identification and controller design are jointly solved.

HIL simulations have been performed to test the performances of the controlled vehicle in a number of different driving conditions: step steer maneuvers on both dry and wet roads, steer reversal test, and side-wind gust disturbance rejection. All the presented maneuvers show that the proposed control system is effective in improving the lateral behavior of the car.
A possible direction for further research is the investigation of the advantages of using virtual sensors inside the loop of a vehicle yaw stability control system. The development of such a technological approach is motivated by recent market requirements aimed at offering low-cost active safety systems. Indeed, a viable solution to reduce costs consists in avoiding the use of physical sensors needed in such control systems. In this context, virtual sensors can be employed to replace hardware ones either to feedback the controlled variables or to obtain redundancy in the achievement of fault tolerance properties.

\section{ACKNOWLEDGMENT}

The authors would like to thank Prof. M. Velardocchia, Dr. A. Morgando, Dr. A. Sorniotti, S. Campo, and A. Fortina for the fruitful discussions during the development of the research.

\section{REFERENCES}

[1] Y. Shibahata, K. Shimada, and T. Tomari, "The improvement of vehicle maneuverability by direct yaw moment control," in Proc. AVEC, Yokohama, Japan, 1992.

[2] S. Inagaki, I. Kshiro, and M. Yamamoto, "Analysis on vehicle stability in critical cornering using phase-plane method," in Proc. AVEC, Tsukuba, Japan, 1994.

[3] S. Matsumoto, H. Yamaguchi, H. Inoue, and Y. Yasuno, "Braking force distribution control for improved vehicle dynamics," in Proc. AVEC 1992, Yokohama, Japan, 1992.

[4] A. V. Zanten, R. Erhardt, and G. Pfaff, "VDC, the vehicle dynamics control system of Bosch," SAE Technical Paper 9507591995.

[5] H. Sato, H. Kawai, and M. Hitisikoike, "Development for four wheel steering system using yaw rate feedback control," in Proc. SAE Tech. Paper Series Passenger Car Meeting Expo., Nashville, TN, Sep. 16-19, 1991.

[6] Y. Shibahata, A. Shimada, K. Shimada, and Y. Furukawa, "Improvement on limit performance of vehicle motion by chassis control," Veh. Syst. Dyn., vol. 23, pp. 449-469, 1994.

[7] Y. Wang, T. Morimoto, and M. Nagai, "Motion control of front-wheel-steering vehicles by yaw moment compensation (comparison with 4WS performance)," Trans. Jpn. Soc. Mech. Eng., vol. 60, no. 571, pp. 912-917, 1994.

[8] M. N. S. Y. Hirano, "Integrated control of active rear wheel steering and yaw moment control using braking forces," JSME Int. J., vol. 42, no. 2, pp. 301-308, 1999.

[9] J. Park and W. Ahn, "H-infinity yaw moment control with brakes for improving driving performance and stability," in Proc. IEEE/ASME Int. Conf. Advanced Intell. Mechatronics, Atlanta, GA, Sep. 19-23, 1999, pp. $747-752$.

[10] B. Güvenç, T. Bünte, D. Odenthal, and L. Güvenç, "Robust two degree-of-freedom vehicle steering controller design," IEEE Trans. Control Syst. Technol., vol. 12, no. 4, pp. 627-636, Jul. 2004.

[11] S. Anwar, "Yaw stability control of an automotive vehicle via generalized predictive algorithm," in Proc. Amer. Control Conf., Portland, OR, Jun. 8-10, 2005, pp. 435-440.

[12] S. Zheng, H. Tang, Z. Han, and Y. Zhang, "Controller design for vehicle stability enhancement," Control Eng. Pract., vol. 14, no. 12, pp. 1413-1421, Dec. 2006.

[13] A. Sorniotti, "Hardware in the loop for braking systems with anti-lock braking system and electronic stability program," SAE Technical Paper 2004-01-2062 2004.

[14] M. Velardocchia and A. Sorniotti, "Hardware-in-the-loop to evaluate active braking systems performance," SAE Technical Paper 2005-0115802005.

[15] R. Rajamani, Vehicle Dynamics and Control. New York: SpringerVerlag, 2006.

[16] H. B. Pacejka, Tyre and Vehicle Dynamics. Warrendale, PA: SAE Int., 2001.

[17] S. Skogestad and I. Postlethwaite, Multivariable Feedback Control. New York: Wiley, 1996.

[18] R. Schrama, "Accurate identification for control: The necessity of an iterative scheme," IEEE Trans. Autom. Control, vol. 37, no. 7, pp. 991-994, Jul. 1992.

[19] P. V. den Hof and R. Schrama, "Identification and control: Closed-loop issues," Automatica, vol. 31, no. 12, pp. 1751-1770, Dec. 1995. 
[20] M. Milanese, M. Taragna, and P. V. den Hof, "Closed-loop identification of uncertainty model for robust control design: A set membership approach," in Proc. 36th IEEE Conf. Decision Control, 1997, pp. 2447-2452.

[21] T. Kevinczky, P. Falcone, F. Borrelli, J. Asgari, and D. Hrovat, "Predictive control approach to autonomous vehicle steering," in Proc. Amer. Control Conf., 2006, pp. 4670-4675.

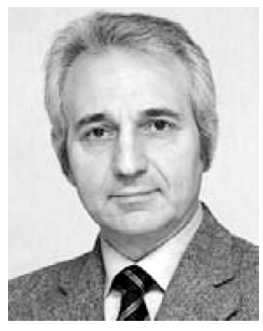

Vito Cerone (M'07) received the Laurea degree in electronic engineering and the Ph.D. degree in system engineering from the Politecnico di Torino, Torino, Italy, in 1984 and 1989, respectively.

In 1989, he spent seven months as an Honorary Research Fellow, supported by a CNR Scholarship, with the School of Electronic and Electrical Engineering, University of Birmingham, Birmingham, U.K. From 1989 to 1998, he was an Assistant Professor with the Dipartimento di Automatica e Informatica, Politecnico di Torino, where he has been an Associate Professor in analysis of dynamical systems, feedback control system design, and analysis and control of physiological systems since 1998. He is a coauthor, with P. Dorato and C. Abdallah, of the book entitled Linear Quadratic Control: An Introduction. He has been involved in the contracts between the Politecnico di Torino and the Centro Ricerche FIAT on "Control strategies for automotive systems" (1993) and "Vehicle lateral and longitudinal dynamics control strategies" (2000). He took part in the contract between the Politecnico di Torino and GM-FIAT Company on "Advanced control strategies for vehicle dynamic control." He has been the Local Coordinator of the research unit of the Politecnico di Torino on the theme "Modelling, identification and control of vehicle lateral and vertical dynamics", which was part of the national project (PRIN2005-Programmi di ricerca di Rilevante Interesse Nazionale): "Control of advanced systems of transmission, suspension, steering and braking for the management of the vehicle dynamics." His main research interests include system identification, parameter estimation, optimization and control, with applications to automotive systems.

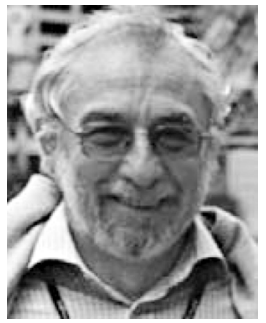

Mario Milanese (SM'04) received the Laurea degree in electronic engineering from the Politecnico di Torino, Torino, Italy, in 1967.

From 1968 to 1972, he was a Teaching Assistant with the Politecnico di Torino, and from 1972 to 1980, he was an Associate Professor of system theory with the Università degli Studi di Torino, Torino. From 1982 to 1987, he was the Head of the Dipartimento di Automatica e Informatica, Politecnico di Torino, where he has been a Full Professor of system theory since 1980 . His research interests include robust identification, prediction and control of uncertain systems, and applications to biomedical, automotive, aerospace, financial and environmental problems. He is the author of more than 180 papers in international journals and conference proceedings. He is the Editor of the books entitled Robustness in Identification and Control (Plenum, 1989) and Bounding Approaches to System Identification (Plenum, 1996).

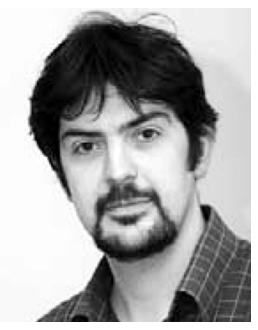

Diego Regruto (M'04) received the Laurea degree in electronic engineering and the Ph.D. degree in system engineering from the Politecnico di Torino, Torino, Italy, in 2000 and 2004, respectively.

He is currently an Assistant Professor with the Dipartimento di Automatica e Informatica, Politecnico di Torino. His main research interests include system identification and robust control, with application to automotive problems. 